\title{
Severe complications and management of a patient with myasthenia gravis undergoing anterior cervical spinal surgery: a case report
}

\author{
Ziquan Li ${ }^{1 \#}$, Muchuan Wang ${ }^{1 \#}$, Cong Zhang ${ }^{2}$, Shujie Wang ${ }^{1}$, Jianguo Zhang ${ }^{1}$, Yipeng Wang ${ }^{1}$, Guixing \\ Qiu $^{1}$, Ye Tian $^{1}$, Siyi Cai ${ }^{1}$ \\ ${ }^{1}$ Department of Orthopedics, Peking Union Medical College Hospital, Chinese Academy of Medical Sciences, Beijing, China; ${ }^{2}$ Department of \\ Endocrinology, China-Japan Friendship Hospital, Beijing, China \\ \#These authors contributed equally to this work. \\ Correspondence to: Siyi Cai, MD; Ye Tian, MD. Department of Orthopedics, Peking Union Medical College Hospital, Chinese Academy of Medical \\ Sciences, No. 1 Shuaifuyuan, Dongcheng District, Beijing 100730, China. Email: drcaisiyi@163.com; pumch_orthopedic@126.com.
}

\begin{abstract}
Surgical management of patients with comorbid long-term myasthenia gravis (MG) is particularly challenging and MG thus represents an independent risk factor for perioperative complications. However, few studies have reported on the perioperative assessment, prevention measures, and risks in MG patients undergoing major surgery, especially for anterior cervical spine surgery. We herein report the rare case of a 62-year-old man with a 20-year history of MG, who was admitted to our hospital with diagnosis of degenerative cervical spondylosis. He safely underwent anterior cervical corpectomy of $\mathrm{C} 4$, discectomy of C5-6, and fusion of C3-6. Intraoperative motor evoked potential was recorded to detect significant improvement after decompression. However, the patient suffered from progressive dysphagia, bucking, and hyperpyrexia 20 days after the initial operation. Imaging revealed titanium cage sliding and graft dislodgement. Secondary surgery was performed for posterior internal fixation from C2-7 and anterior revision from C3-6 after Halo-Vest traction, antibiotic treatment, and immunoglobulin therapy. He underwent a series of postoperative treatments, including cervicothoracolumbosacral orthosis, atomization inhalation, chest physiotherapy, antibiotics, and nutritional support. His condition improved markedly and he had no recurrence of symptoms during the 6-month follow-up. It is the rare reported case of anterior cervical spinal surgery in a patient with MG. This rare case indicates a relative contraindication to anterior-only approaches especially with multiple levels for MG patients with cervical spondylosis. Posterior approach, intraoperative monitoring, osteoporosis, postoperative strong brace protection, and supportive management should be considered for patients who were on large doses of steroids for long duration of time, given the lack of sufficient bone mineral density.
\end{abstract}

Keywords: Myasthenia gravis (MG); anterior cervical surgery; case report; dysphagia; internal fixation failure

Submitted Mar 24, 2021. Accepted for publication May 21, 2021.

doi: 10.21037/apm-21-695

View this article at: https://dx.doi.org/10.21037/apm-21-695

\section{Introduction}

Myasthenia gravis (MG) is caused by antibodies binding to acetylcholine receptors in the postsynaptic membrane at the neuromuscular junction, which is characterized by blepharoptosis, skeletal muscle weakness, fatigability, and dysphagia $(1,2)$. Surgery represents a major challenge in patients with MG because of the etiologies and clinical features of the disease, including exacerbated muscle weakness, pneumonia, septicemia, and myasthenia crises $(3,4)$. However, few studies have reported on MG patients undergoing major surgery and there have been only two reports of patients presented with MG after 


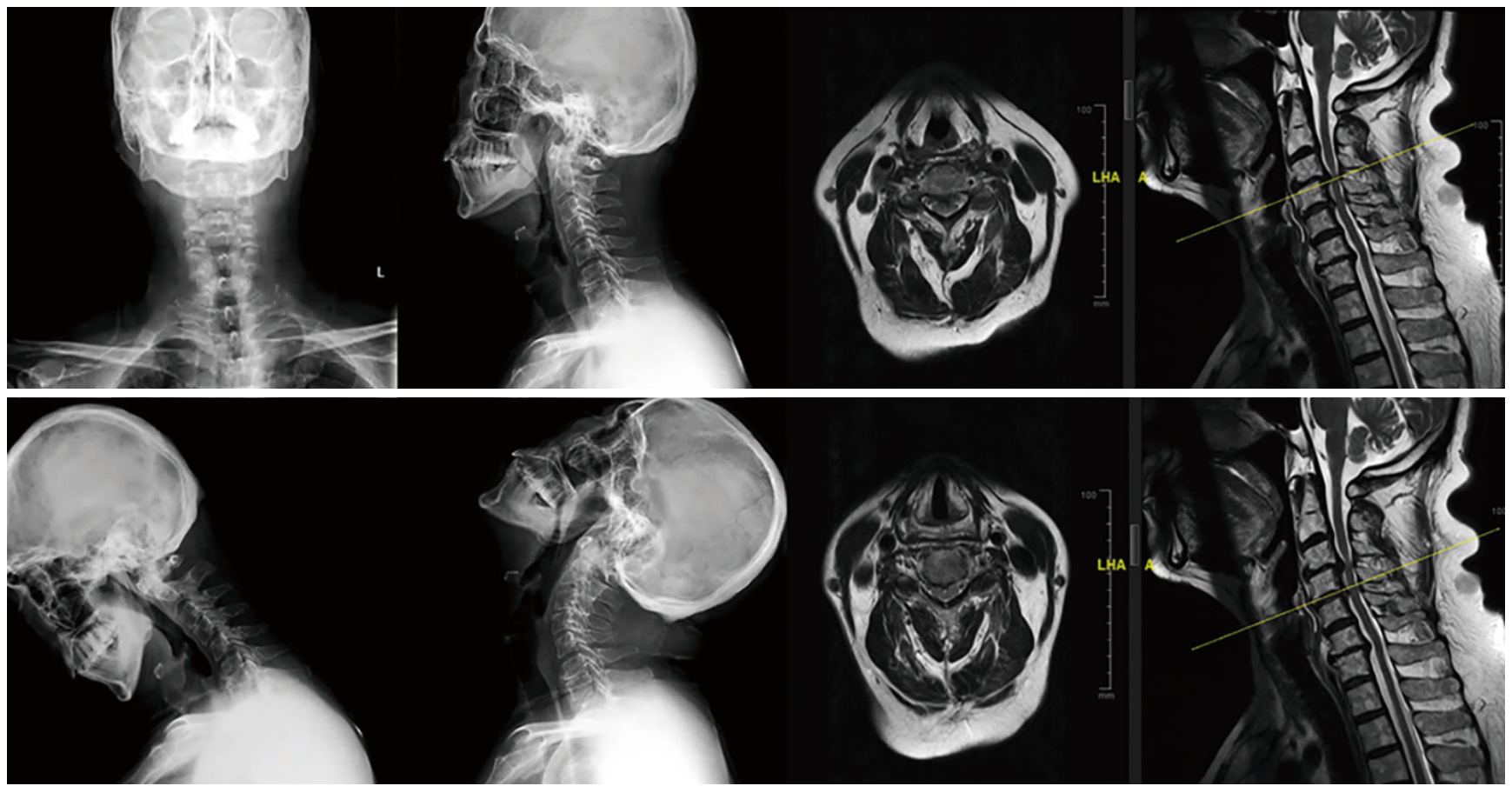

Figure 1 Preoperative imaging features in a myasthenia gravis patient with cervical spondylosis and cervical spondylotic myelopathy. X-ray showed degenerative changes and facet joint hyperplasia of the cervical spine. Sagittal and axial T2-weighted magnetic resonance images of the cervical spine showed compression of spinal cord at C3-4, C4-5, and C5-C6.

cervical spine surgery $(5,6)$. We therefore clarified the postoperative complications and analyzed the most likely etiologies, possible preventive measures, and recommended therapies in a rare case of patient comorbid long-term MG undergoing anterior cervical spinal surgery. We present the following case in accordance with the CARE reporting checklist (available at https://apm.amegroups.com/article/ view/10.21037/apm-21-695/rc).

\section{Case presentation}

\section{Clinical history}

A 62-year-old man was admitted to Peking Union Medical College Hospital with complaints of unstable gait, progressively worsening hand dexterity, and numbness in both upper limbs. Physical examination revealed reduced muscle bulk, increased muscle tone in bilateral lower limbs and bilaterally positive of Hoffman sign and Chaddock's sign. Motor examination evaluated his bilateral upper extremity strength as grade $4 / 5$ according to the British Medical Research Council scale. Hand-grip strength was $15 \mathrm{~kg}$ on the left and $20 \mathrm{~kg}$ on the right side. X-ray and magnetic resonance imaging demonstrated degenerative cervical spondylosis (Figure 1).

The patient also suffered from prominent ptosis, limb weakness, hoarseness combined with dysphagia, and had been diagnosed with MG (Osserman IIb) by a neurologist 20 years previously, according to the following indications: confirmed by muscle biopsy, diminished response with low frequency stimuli by repetitive nerve stimulation, elevated acetylcholine receptor antibody levels (presynaptic membrane 1.23 , postsynaptic membrane 1.06 ), positive neostigmine test, thymic hyperplasia with no enhancement on enhanced computed tomography scan of the chest, and no significant findings on positron emission tomography. He had been treated with oral pyridostigmine bromide (60 mg tid) and methylprednisolone 1,000 $\mathrm{mg}$ qd for 3 days initially, and then changed to oral prednisone $60 \mathrm{mg} \mathrm{qd}$. The usage and dosage of the corticosteroid were gradually reduced to oral methylprednisolone $8-12 \mathrm{mg}$ qd under the guidance of the neurologist in the recent one year. He had also received intravenous immunoglobulin (IVIG) $0.4 \mathrm{~g} / \mathrm{kg} /$ day for 5 days each year for the past 8 years. His symptoms of myasthenia were considered to be satisfactorily controlled after the 

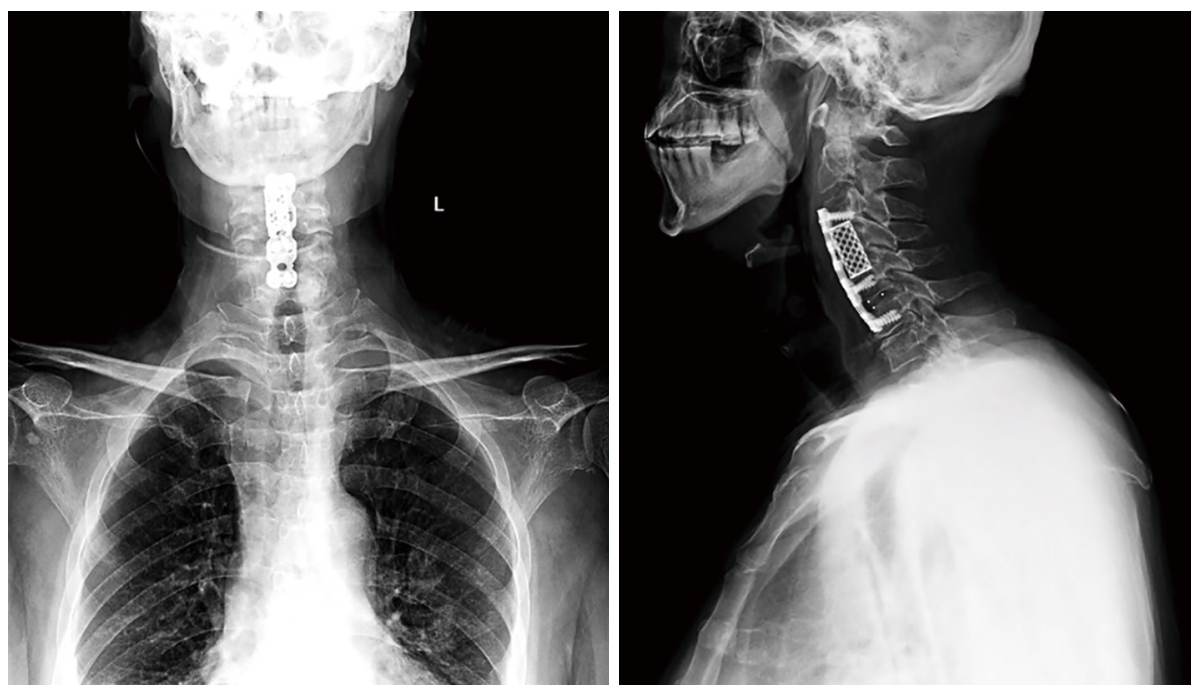

Figure 2 Postoperative X-ray features of cervical spine after initial surgery. X-ray was performed 2 days after initial surgery. AP and lateral films illustrated anterior C4 corpectomy, C5-6 discectomy, internal fixation and fusion from C3-6 with good alignment of the construct. AP, anterior-posterior.

treatment with IVIG. He had medication history of azathioprine and cyclophosphamide, but the medication was discontinued because there was no obvious effect. Preoperative pulmonary function test demonstrated obstructive ventilation function disturbance with forced expiratory volume in the first second of exhalation (FEV1) $1.66 \mathrm{~L}$ (FEV1\% 45.6\%) and forced vital capacity (FVC) $2.51 \mathrm{~L}$.

\section{Initial surgery and motor evoked potential (MEP) monitoring}

According to a neurology consultation, the patient received $30 \mathrm{~g}$ IVIG 5 days before surgery. He then underwent C4 corpectomy, C5-6 discectomy, decompression, internal fixation with autologous bone grafting from $\mathrm{C} 3-6$, and titanium cage and Bengal cage implantation by a standard right-sided anterior cervical approach (Depuy, USA). No muscle relaxants or inhalation agents were given after anesthesia induction and intubation.

Intraoperative MEP was elicited by subcutaneous needle electrodes after stimulation with a constant voltage (250-500 V) and multiple trains of six to seven pulses (Axon Systems Inc., Hauppauge, NY, USA). MEPs were recorded to detect significant improvement after decompression. The left-side signal increased from 30 to $100 \mu \mathrm{V}$, and the right-side from 120 to $300 \mu \mathrm{V}$.

He was transferred to the surgical intensive care unit postoperatively, for close observation of muscle fatigue or cholinergic crisis. The patient was extubated and transferred to the orthopedic department on the first postoperative day. The patient's initial recovery was uneventful, and his symptoms improved. A postoperative $\mathrm{X}$-ray of his cervical spine was shown in Figure 2. He was discharged home 3 days after surgery with Philadelphia cervical orthosis.

\section{Postoperative complications}

At 20 days after the initial surgery, the patient was admitted to the emergency department complaining of gradually worsening dysphagia, bucking, cough with yellow phlegm, and fever. Biochemical examination revealed an increased erythrocyte sedimentation rate, C-reactive protein, and a high white blood cell count of $16.11 \times 10^{9} / \mathrm{L}$ with neutrophilia. These results combined with chest X-ray results suggested the possibility of an acute pulmonary infection. Anterior-posterior (AP) and lateral X-ray images of the cervical spine demonstrated vertebral fracture, implant extrusion, and cage sliding (Figure 3).

\section{Interventions}

The patient's complaints, clinical signs, and radiological characteristics suggested implant failure. He was initially treated with Halo-vest traction to reduce and stabilize his cervical spine (Figure 4). His infection was controlled by 

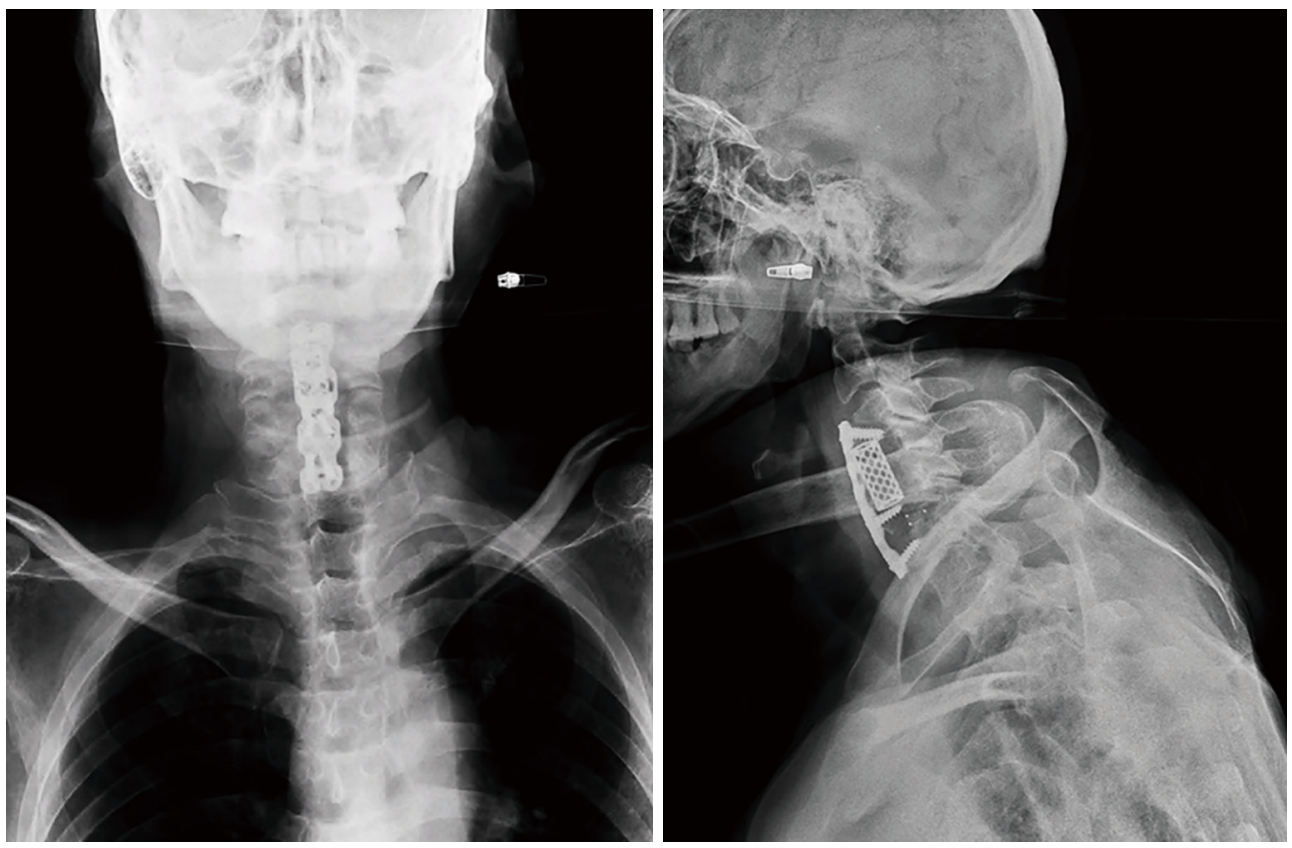

Figure 3 Typical X-ray of implant extrusions following anterior cervical surgery. Vertebral compression fracture of C3 and C5, graft migration, plate dislodgment and cage sliding were shown in AP and lateral views on X-ray. AP, anterior-posterior.
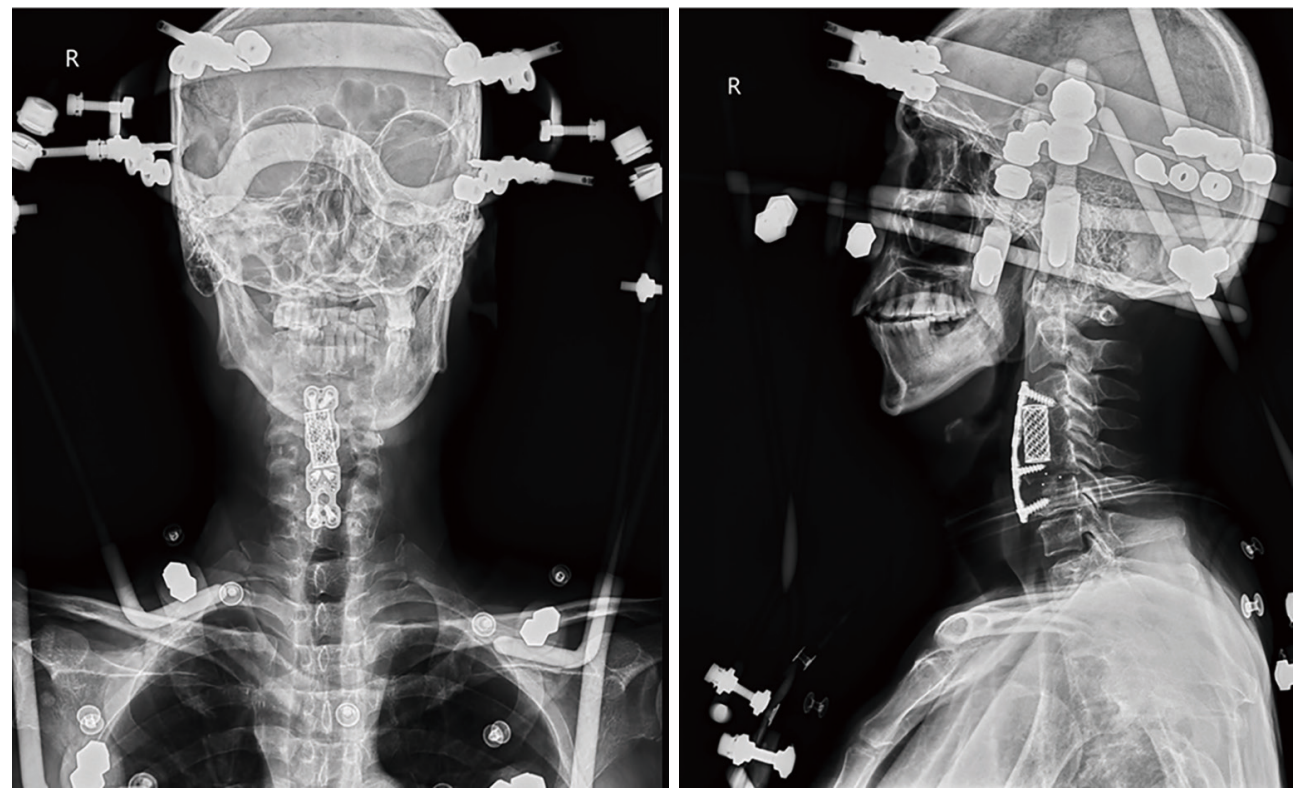

Figure 4 Halo-vest traction was performed 1 month after initial surgery. AP and lateral X-ray images depicted that the cervical spine was stable and alignment of cervical spine was partially improved after halo-vest traction. AP, anterior-posterior.

cefuroxime (1.5 g, q12h for 14 days intravenous), followed by posterior and anterior revision surgery. Posterior internal fixation and bone graft fusion from $\mathrm{C} 2-7$ were performed first, followed by anterior revision surgery. The previously inserted plate and screws were seen to be loose, combined with displacement of the cage. No cerebrospinal 


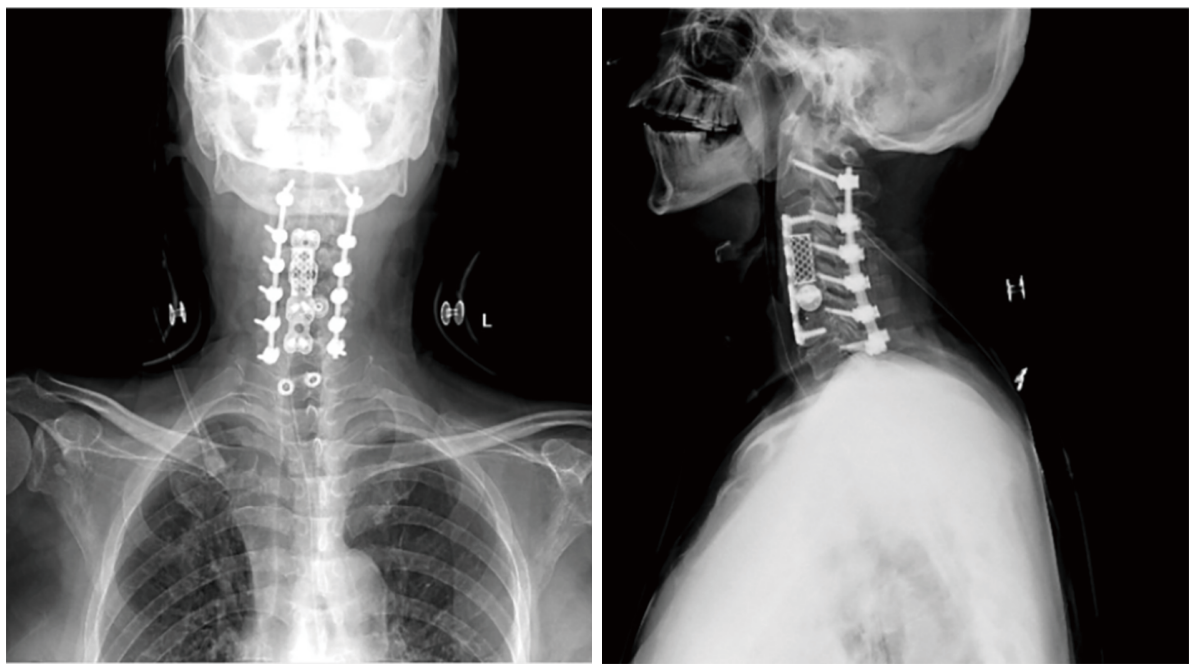

Figure 5 Postoperative X-ray at 3 months' follow-up after revision surgery. Postoperative AP and lateral X-ray images showed good alignment of the implant after anterior revision and posterior instrumentation at C2-C7. AP, anterior-posterior.

fluid leakage was seen during surgery and MEP monitoring showed no nerve deficits.

\section{Postoperative course}

The patient developed recurrent pneumonia and malnutrition as a result of postoperative dysphagia. A series of supportive treatments, including a Milwaukee brace, long-term antibiotic therapy, respiratory management, and enteral nutrition were performed, and the patient recovered well. Close follow-up was performed at 3 and 6 months after the revision surgery. The patient's symptoms improved, with a good mental state, appetite, and physical strength. The Japanese Orthopedic Association (JOA) score was 15 at the last follow-up, compared with that was 8 preoperatively. A follow-up X-ray showed satisfactory positioning of the internal fixation and a stable cervical spine (Figure 5). All procedures performed in this study were in accordance with the ethical standards of the institutional and national research committee(s) and with the Helsinki Declaration (as revised in 2013). Written informed consent was obtained from the patient for publication of this case report and accompanying images. A copy of the written consent is available for review by the editorial office of this journal.

\section{Discussion}

We provide the rare case report of a patient with long-term MG who underwent an anterior cervical spine surgery for cervical spondylosis. The present patient's MG was stably maintained and we avoided the use of muscle relaxants after anesthesia induction and intubation. However, our patient suffered from disease-related complications after surgery. Although no definitive etiology was determined, the patient's characteristics suggested that long-term MG and glucocorticoid induced osteoporosis might have led to graft failure during the early postoperative period in this case.

MG is an autoimmune neuromuscular disease that affects the bulbar muscles, causing dysphagia, dysarthria, and dyspnea. Dysphagia may lead to aspiration of saliva, food or liquids, resulting in several medical complications, such as malnutrition, aspiration pneumonia, and even respiratory failure $(7,8)$. Furthermore, postoperative dysphagia is one of the most common complications after anterior cervical spine surgery as a result of surgical trauma, mucosal edema, and esophageal damage (9-11). Because of the comorbidity of MG and stress of anterior cervical surgery, the current patient in our case report complained of progressive dysphagia postoperatively, accompanied by reflexive coughing during or right after swallowing. Moreover, recurrent dysphagia, weak cough, and an inability to expel secretions may result in respiratory aspiration, potentially increasing the risk of recurrent pneumonia postoperatively $(12,13)$. Importantly, reflexive coughing, respiratory aspiration, and recurrent inflammation after anterior cervical spine surgery are significant risk factors for internal fixation failure in the early postoperative period $(14,15)$. In addition, $M G$ 
patients also suffer from more opportunistic infections because of systemic inflammatory course of MG and their immunocompromised status, associated with long-term use of glucocorticoids or immunosuppressive agents $(16,17)$.

Multiple risk factors contribute to osteoporosis and increase the incidence of fracture in $\mathrm{MG}$, including chronic muscle weakness, physical inactivity, lack of sunlight exposure, vitamin D deficiency, comorbidity with thyroid disorder, and treatment with glucocorticoids (18). Although glucocorticoids are currently the first-line treatment for $\mathrm{MG}$, osteoporosis with consequent bone fractures is one of the most common and severe adverse effects of glucocorticoids (19). Osteoporosis is another major cause of implant failure after spinal surgery. A retrospective study of patients with degenerative cervical disease indicated that patients with osteoporosis were more likely to undergo revision surgery after cervical spinal procedures (20). Our patient received long-term glucocorticoid therapy and demonstrated severe osteoporosis. We therefore speculated that glucocorticoid-induced osteoporosis decreased the screw pull-out strength and fatigue resistance capability, resulting in pedicle screw instability and implant dislodgment, which may be another contributory factor to instrumentation failure during the early postoperative period.

The proposed etiology in the current case suggested that the following procedures may help to prevent perioperative complications in MG patients undergoing cervical spine surgery. First, intraoperative MEP monitoring is recommended to increase the safety of cervical surgery. The current case indicates that the intraoperative MEP would not be affected in patient with MG during spinal surgery. Additionally, our experience suggests that MG patients with cervical spondylosis are more likely to have disease-related complications postoperatively. It is therefore critical to make the decision to perform anterior cervical surgery cautiously. Postoperative protection with a neck brace, and use of a posterior, or anterior combined with posterior approach should be considered to provide more support and reduce the risk of graft migration. Thirdly, preoperative bone mineral density measurements using dual-energy $\mathrm{X}$-ray absorptiometry and treatment of osteoporosis should be suggested.

In conclusion, patient with cervical spondylosis and comorbid long-term MG can result in unusual postoperative complications after anterior cervical spinal surgery. This rare case reminds that it should be very cautious of going anteriorly for patients with MG or patients taking large doses of steroids for long durations of time.

\section{Acknowledgments}

The authors would like to thank the patient and his family members for their help and informed consent.

Funding: This work was supported by China Postdoctoral Science Foundation (No.2020TQ0052) and the National Natural Science Foundation of China (Grant No. 81871746).

\section{Footnote}

Reporting Checklist: The authors have completed the CARE reporting checklist. Available at https://apm.amegroups. com/article/view/10.21037/apm-21-695/rc

Conflicts of Interest: All authors have completed the ICMJE uniform disclosure form (available at https://apm. amegroups.com/article/view/10.21037/apm-21-695/coif). The authors have no conflicts of interest to declare.

Ethical Statement: The authors are accountable for all aspects of the work in ensuring that questions associated with the accuracy or integrity of any part of the work are appropriately investigated and resolved. All procedures performed in this study were in accordance with the ethical standards of the institutional and national research committee(s) and with the Helsinki Declaration (as revised in 2013). Written informed consent was obtained from the patient for publication of this case report and accompanying images. A copy of the written consent is available for review by the editorial office of this journal.

Open Access Statement: This is an Open Access article distributed in accordance with the Creative Commons Attribution-NonCommercial-NoDerivs 4.0 International License (CC BY-NC-ND 4.0), which permits the noncommercial replication and distribution of the article with the strict proviso that no changes or edits are made and the original work is properly cited (including links to both the formal publication through the relevant DOI and the license). See: https://creativecommons.org/licenses/by-nc-nd/4.0/.

\section{References}

1. Gilhus NE. Myasthenia Gravis. N Engl J Med 2016;375:2570-81.

2. Fortin E, Cestari DM, Weinberg DH. Ocular myasthenia gravis: an update on diagnosis and treatment. Curr Opin 
Ophthalmol 2018;29:477-84.

3. Alshaikh JT, Amdur R, Sidawy A, et al. Thymectomy is safe for myasthenia gravis patients: Analysis of the NSQIP database. Muscle Nerve 2016;53:370-4.

4. Keijzers M, de Baets M, Hochstenbag M, et al. Robotic thymectomy in patients with myasthenia gravis: neurological and surgical outcomes. Eur J Cardiothorac Surg 2015;48:40-5.

5. Deters D, Fowler SL, Orozco R, et al. Myasthenia Gravis Presentation After a Cervical Laminectomy With Fusion. Dimens Crit Care Nurs 2016;35:190-4.

6. Mangal V, Rajagopal A, Rothenberg DM. Unusual presentation of myasthenia gravis after cervical spine surgery. Int Anesthesiol Clin 2011;49:71-3.

7. Shaw SM, Martino R. The normal swallow muscular and neurophysiological control. Otolaryngol Clin North Am 2013;46:937-56.

8. Britton D, Karam C, Schindler JS. Swallowing and secretion management in neuromuscular disease. Clinics in Chest Medicine 2018;39:449-57.

9. Kalb S, Reis MT, Cowperthwaite MC, et al. Dysphagia after anterior cervical spine surgery: incidence and risk factors. World Neurosurg 2012;77:183-7.

10. Liu JM, Tong WL, Chen XY, et al. The incidences and risk factors related to early dysphagia after anterior cervical spine surgery: a prospective study. PLoS One 2017;12:e0173364.

11. Singh K, Marquez-Lara A, Nandyala SV, et al. Incidence and risk factors for dysphagia after anterior cervical fusion.
Spine (Phila Pa 1976) 2013;38:1820-5.

12. Britton D, Karam C, Schindler JS. Swallowing and Secretion Management in Neuromuscular Disease. Clin Chest Med 2018;39:449-57.

13. Termsarasab P, Katirji B. Opportunistic infections in myasthenia gravis treated with mycophenolate mofetil. J Neuroimmunol 2012;249:83-5.

14. Fineberg SJ, Oglesby M, Patel AA, et al. Incidence, risk factors, and mortality associated with aspiration in cervical spine surgery. Spine (Phila Pa 1976) 2013;38:E1189-95.

15. Konopka JA, Armaghani SJ, Dao AM, et al. Superior Laryngeal Nerve Palsy in Revision Anterior Cervical Surgery. J Am Acad Orthop Surg 2020;28:e181-4.

16. Gilhus NE, Nacu A, Andersen JB, et al. Myasthenia gravis and risks for comorbidity. Eur J Neurol 2015;22:17-23.

17. Fang F, Sveinsson O, Thormar G, et al. The autoimmune spectrum of myasthenia gravis: a Swedish populationbased study. J Intern Med 2015;277:594-604.

18. Guan Y, Lv F, Meng Y, et al. Association between bone mineral density, muscle strength, and vitamin $\mathrm{D}$ status in patients with myasthenia gravis: a cross-sectional study. Osteoporos Int 2017;28:2383-90.

19. Braz NFT, Rocha NP, Vieira ÉLM, et al. Negative impact of high cumulative glucocorticoid dose on bone metabolism of patients with myasthenia gravis. Neurol Sci 2017;38:1405-13.

20. Guzman JZ, Feldman ZM, McAnany S, et al. Osteoporosis in Cervical Spine Surgery. Spine (Phila Pa 1976) 2016;41:662-8.
Cite this article as: $\mathrm{Li} Z$, Wang M, Zhang $\mathrm{C}$, Wang S, Zhang J, Wang Y, Qiu G, Tian Y, Cai S. Severe complications and management of a patient with myasthenia gravis undergoing anterior cervical spinal surgery: a case report. Ann Palliat Med 2022;11(4):1561-1567. doi: 10.21037/apm-21-695 